\title{
Timing of Surgery and Clinical Outcomes of Isolated Right-heart Infective Endocarditis. A Single Center Experience
}

\author{
(1) Ahmet Feyzi Abacılar
}

İzmir Private Su Hospital, Clinic of Cardiovascular Surgery, İzmir, Turkey

\begin{abstract}
Objectives: Isolated infective endocarditis of the right heart is a rare clinical entity. I review our twelve-year experiences of the peri-operative features and surgical treatment of isolated right-sided infective endocarditis and long-term outcomes.
\end{abstract}

Materials and Methods: Between January 2005 and June 2017, a total of 58 patients were operated for an isolated right-sided infective endocarditis in our tertiary center. Congenital heart defects were the main reasons. Previous pacemaker lead insertion, cardiac catheterization, use of central vein catheters for hemodialysis, and intravenous drug abuse were other risk factors. Seven patients have a history of active intravenous drug use (12\%). The patients' mean follow-up was $24.7 \pm 6.1$ months.

Results: Three patients died after surgery (5.1\%) due to postoperative low out-put syndrome and uncontrolled septic shock after surgery. Two patients had chronic kidney disease and one used intravenous drug. Tricuspid valve repair or replacement was performed in 29 patients $(50 \%)$. Mechanical and bioprosthetic heart valves were replaced in eight patients (13.7\%). De Vega, Kay's annuloplasty, or bicuspidization were performed in 21 patients (36.2\%). Coagulase-negative staphylococci ( $\mathrm{n}=14)$, Staphylococcus epidermidis $(\mathrm{n}=7)$, Streptococcus viridans $(\mathrm{n}=5), S$. epidermidis $(\mathrm{n}=3)$, and Methicillin Resistance Staphylococcus aureus $(\mathrm{n}=3)$ were the most common microorganisms in pre-operative blood cultures. In the patients, who had a history of intravenous drug abuse ( $\mathrm{n}=7)$, Enterococcus and fungi were the pathogens. Two patients $(3.8 \%)$ required re-operation because of the recurrence of endocarditis, combined with severe tricuspid valve impairment seven months and one year after the first operation. The survival rate after operation was 30 days, and $1,2,5$, and 10 years $(94.2 \%, 88.9 \%, 82.5 \%, 81 \%$, and $80 \%)$, respectively.

Conclusion: Surgical outcomes of patients with isolated right-sided infective endocarditis, who underwent surgery in the early time, were favourable. We suggest extensive and an aggressive intervention when the patient have hemodynamic instability or the right heart failure which is resistant to medical treatment or large vegetation. Postoperative antibiotic treatment and medication are the key factors to avoid mortality and morbidity.

Keywords: Isolated right heart infective endocarditis, surgery, congestive heart failure

Address for Correspondence: Ahmet Feyzi Abacılar, İzmir Private Su Hospital, Clinic of Cardiovascular Surgery, İzmir, Turkey Phone: +90 5334813056 e-mail: afeyziabacilar@gmail.com ORCID: orcid.org/0000-0002-9483-4035 Received: 17.09.2019 Accepted: 31.10 .2019

Cite this article as: Abacılar AF. Timing of Surgery and Clinical Outcomes of Isolated Right-heart Infective Endocarditis. A Single Center Experience. EJCM 2019;7(4):179-188.

DOI: $10.32596 /$ ejcm.galenos.2019.09.050

${ }^{\circ}$ Copyright 2019 by Heart and Health Foundation of Turkey (TÜSAV) / E Journal of Cardiovascular Medicine published by Galenos Publishing House. 


\section{Introduction}

The isolated right-sided infective endocarditis (IRSIE) of the right heart is a rare but serious clinical condition. About $70 \%$ of patients with IRSIE may be treated conservatively without the need of surgery ${ }^{(1-4)}$. According to current recommendation of medical or surgical approach in a small number of case series is vary. Persistent infection despite antibiotic therapy, recurrent pulmonary embolism and tricuspid valve impairment with heart failure are the most common indications in these patients ${ }^{(3-6)}$. Septic shock due to IRSIE, the vegetation size (more than $>2 \mathrm{~cm}$ ), or acute renal or hepatic failure are accepted as other indications for surgery. The authors proposed the early surgical approach in patient with severe valve destruction combined with annular damage because of the risk of morbidity and mortality despite medical management ${ }^{(7)}$. There are small number of clinical investigations and surgical outcomes in the literature ${ }^{(1,2)}$. Tricuspid valve is affected in some of the patients with $\operatorname{IRSIE}^{(3)}$. The main risk factors of IRSIE are congenital heart disease, degenerative cardiac valvular disease, central venous catheter use in patients with chronic kidney disease, and a pacemaker lead ${ }^{(4,5)}$. Intravenous drug abuse is a rare but severe reason in patients with IRSIE, which is reported in a small number of patients in the literature ${ }^{(8)}$. Extensive vegetatectomy in combination with an intracardiac (peri-annular) abscess, artificial valve replacement, or a valvectomy of tricuspid valve have been suggested ${ }^{(7-10)}$. In addition, because the use of intracardiac non-biologic material can increase the recurrence of IRSIE, the authors have suggested a biologic material in the surgical treatment ${ }^{(11-13)}$.

Herein, we present the surgical approaches and clinical outcomes of 58 patients with IRSIE without the involvement of the left heart and long-term follow-up after surgery.

\section{Materials and Methods}

The 38 male and 20 female patients have been operated for isolated IRSIE in our tertiary center. The erythrocyte sedimentation rates and C-reactive protein levels were high in all patients. There was moderate anemia in 16 patients (hemoglobin levels were between 7.6 and $9.1 \mathrm{mg} / \mathrm{dL}$ ). The patients' ages ranged from 16 to 67 years (mean ag: 32.7 years). All patients were admitted with persistent fever, intractable heart failure, uncontrollable sepsis, or large vegetations. The clinical characteristics of the patients have been summarized in Table 1. The time from the manifestation of clinical symptoms to diagnosis ranged from six days to three weeks. The reasons for endocarditis were congenital heart defects, pacemaker lead insertion, intravenous drug use (IVDU), right-sided catheterization for hemodialysis, and previous cardiac surgery. Pre-operatively, 33 patients were in New York Heart Association (NYHA) functional class I or II, and 25 were in class III-IV.

A transthoracic or transesophageal echocardiogram revealed intracardiac vegetation(s) in all patients (Figure 1). Intracardiac thrombus formation was detected in 13 patients (Figure 2). There were positive blood cultures in 50 patients $(86.2 \%)$. In accordance with this definition, 52 patients had an active IRSIE. Pre-operative chest radiographs showed pulmonary infections in 17 patients (34.6\%). Pulmonary embolism was detected using a thoracic computed tomography in three IVUS patients (5.7\%) (Figure 3 ). The vegetation diameter exceeded $20-29 \mathrm{~mm}$ in 21 patients. In nine patients, the diameter of vegetations was $33-41 \mathrm{~mm}$. Twenty-nine patients required tricuspid valve repair $(\mathrm{n}=12 ; 20.6 \%)$ or replacement $(17 ; 29.3 \%)$ because of serious valvular damage, valvular impairment (degree 3 or more), intracardiac thrombus or peri-annular abscess formation. Moderate impairment of tricuspid valve was detected in eight patients.

According to the blood culture, we started antibiotics and medical management for 4-6 weeks in the intensive care units. The mean duration of antibiotics usage was $36.8 \pm 11.4$ days. The main indications for surgery of our patients were intractable cardiac failure, intractable sepsis, recurrent pulmonary embolic events, large or mobile vegetation(s), and intracardiac thrombus formation. 
In seven IVDUs, the etiology of IRSIE was fungi enterococcus, except in one patient. The location of IRSIE and predominant microorganisms and surgical procedures (the type of repair technique or replacement) have been summarized in Table 2. Pulmonary artery hypertension was detected in 26 patients $(44.8 \%)$. The mean rate of left ventricular ejection fraction was $43.8 \pm 9.4 \%$. We performed hemodialysis prior to cardiac surgery in 11 patients $(12 \%)$. Three IVDUs required a temporary renal replacement therapy after surgery in the ICU.

Table 1. Pre-operative patients' charecteristics of both groups

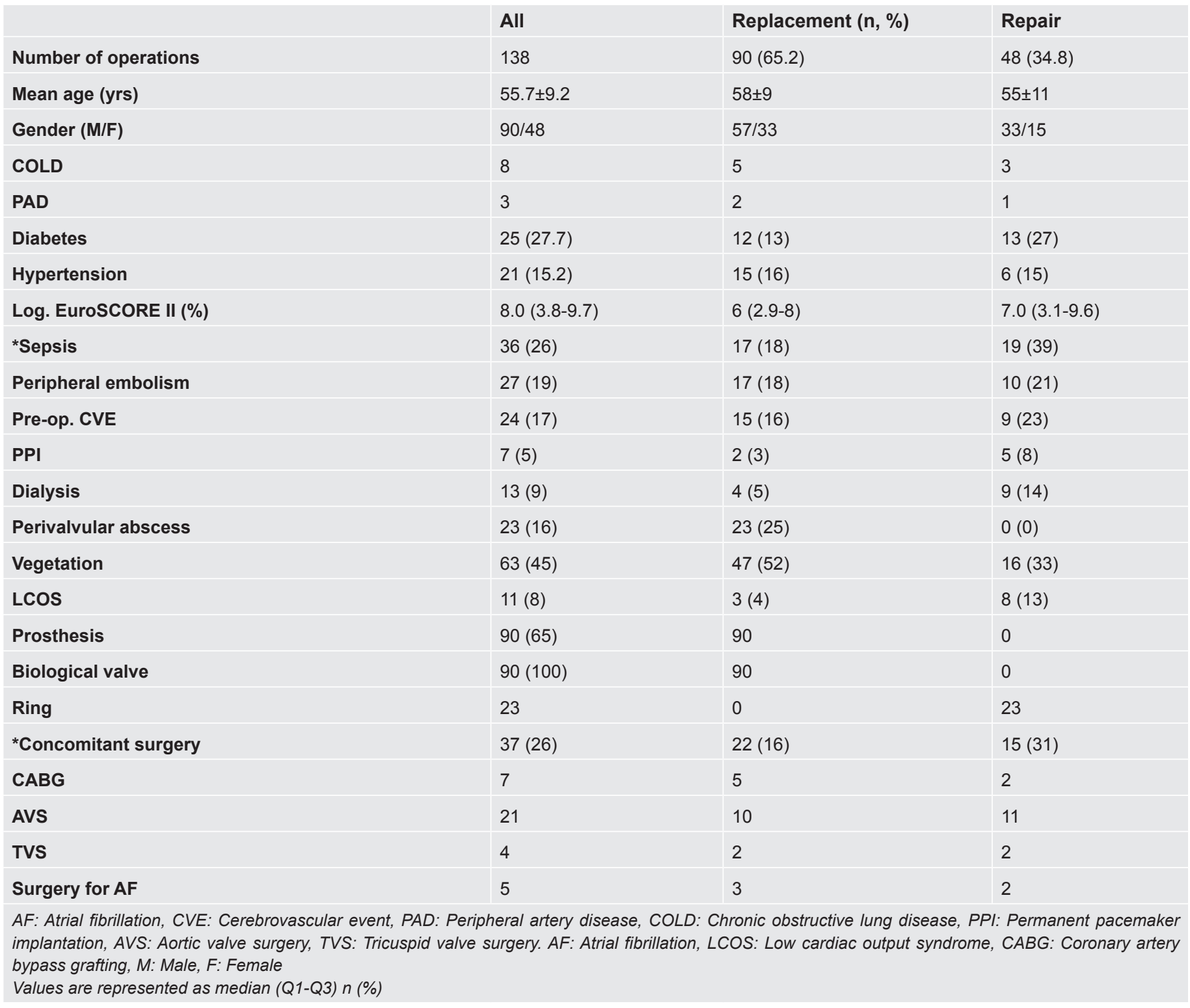

There is no necessary to obtain ethics committee approval and informed consent due to the study is retrospective.

\section{Surgical Approaches}

After a median sternotomy incision, an aortic and bicaval cannulation was performed. Cardiopulmonary bypass was instituted without the handling of the heart to provide embolic events. Cardiac arrest has been provided by the use of antegrade cold blood cardioplegia 
via ascending aorta. The aim of our surgical strategy is based on principles of (a) intensive debridement of the infected area including prosthetic material followed by vegetectomy, (b) if possible, performance of the tricuspid valve repair without the use of prosthetic material, and (c) if valve replacement is unavoidable, use of a biological substitute without any artificial material. Following these strategies, we achieved good clinical results without the recurrence in the early, mid-term and long-term followups.

We repaired using a pericardium or biologic materials in patients with concomitant congenital cardiac defect after an intensive debridement. If the reason of IRSIE was a lead of pacemaker, the lead was removed carefully, and vegetations were cleared extensively. To prove arrhythmia or atrioventricular block, an intensive debridement of the infected cardiac chambers was performed with careful attention.

Of the patients with severely degenerative tricuspid valve and adjacent tissue due to endocarditis, we excised the tricuspid valve only in three patients. We replaced an artificial biologic heart valve. In appropriate patients,

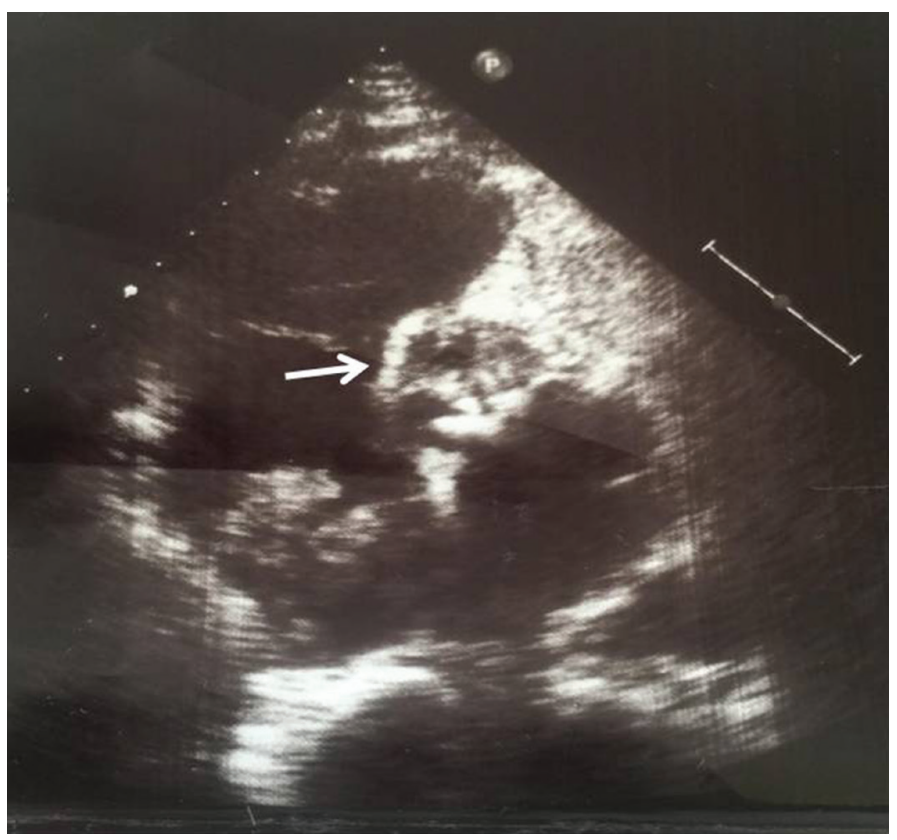

Figure 1. Transthoracic echocardiography shows the large vegetation in a female patient (white arrow) we reconstructed tricuspid valve directly or using an autologous pericardium (Figure 4). Leaflet-plasty, De Vega or Kay's annuloplasty, or bicuspidization were performed in 18 patients. To provide suitable leaflet coaptation, bioprosthetic annuloplasty rings were used. We detected pulmonary valve destruction due to endocarditis in four patients with intravenous drug abuse. We used a pericardial patch to reconstruct the pulmonary valve after vegetation removal. Complications after procedures and redo-operation in both groups are summarized in Table 3.

The patients were weaned from ECC successfully. To provide hemodynamic stability, we used inotropic agent(s) in 32 patients. The broad-spectrum antibiotics were administered for 4 to 6 weeks according to the results obtained from blood cultures. In patients with IRSIE, who underwent hemodialysis, hemofiltration was performed during the cardiac surgery.

\section{Statistical Analysis}

Statistical analyses were performed with SPSS software version 19.0. Continuous data were summarized as mean \pm standard deviation or median with interquartile range $\left(25^{\text {th }}-75^{\text {th }}\right.$ percentiles $)$, and categorical data were summarized as percentages or frequencies. Differences

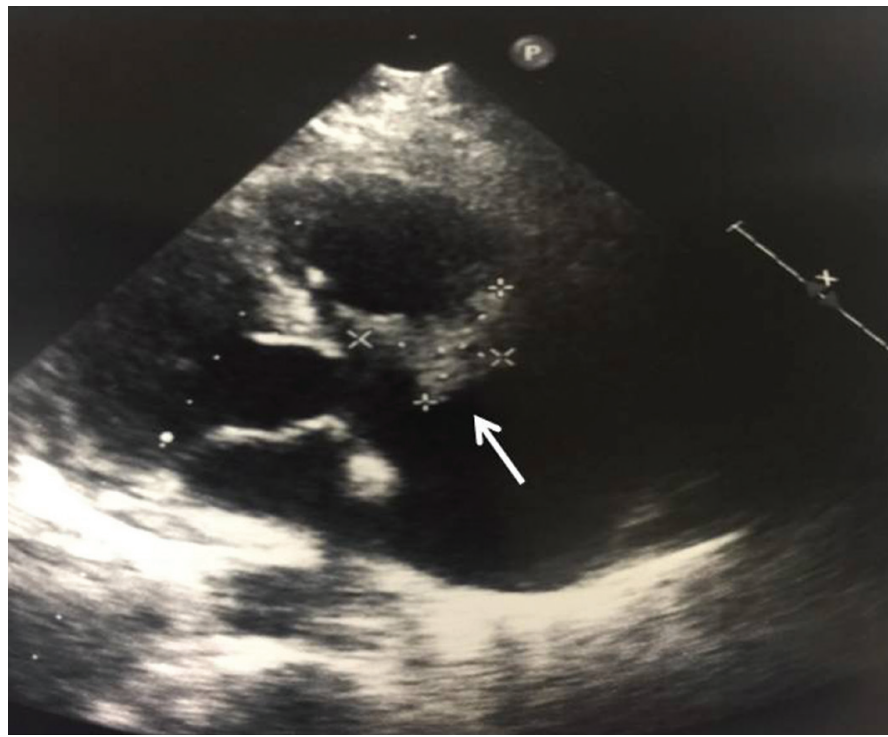

Figure 2. Transthoracic echocardiography demonstrates the right heart thrombus and vegetations in a male patient who underwent atrial septal defect closure previously (white arrow) 
between repair and replacement groups were compared with the use of a Fisher's exact test for categorical variables and the Wilcoxon rank-sum test for the continuous variables. $\mathrm{P}$ values of $<0.05$ were considered as statistically significant.

\section{Results}

In 50 samples of excised vegetation, the results were positive for microorganisms. By using a microscopy, we diagnosed seven IVDUs to have fungal endocarditis and Enterococcus. The species of fungi were Candida albicans, Aspergillus fumigatus, and Fusarium. In these patients, we administered Voriconazole, which has a wide spectrum of antifungal agent. Amphotericin-B was given at higher doses in the management of Aspergillus because it was less toxic than the conventional amphotericin in a patient with kidney dysfunction or developing nephrotoxicity while receiving classic amphotericin. Itraconazole and caspofungin were administered as an effective drug of refractory Aspergillus infection. Transesophageal echocardiography showed no tricuspid regurgitation in 13 patients who underwent tricuspid valvuloplasty. Of 18 patient who underwent valvuloplasty, two had moderate regurgitation.

Three patients died because of uncontrollable sepsis, and $\operatorname{LOS}(\mathrm{n}=5.1)$. In the repair group, two patients required revision because of postoperative bleeding (3.3 $\%)$. Ventilation assistance was provided for more than 4 days in seven patients with NYHA Class III-IV. The new onset of acute renal failure developed in three IVDUs who required temporary hemodialysis $(5.1 \%)$. The mean length of stay in the ICU was $5.7 \pm 1.6$ days. Atrioventricular block was detected in three patients. Therefore, permanent pacemakers were implanted 2 weeks later.

\section{Follow-up Period}

Three patients died because of unknown reason in the follow-up period. We followed 39 patients using physical examination and echocardiography in our patient clinic. In the remaining patients, data were provided through

Table 2. Intra-operative and postoperative properties of both groups

\begin{tabular}{|c|c|c|c|}
\hline & Total & Replacement & Repair \\
\hline Number of operations & 138 & 90 & 48 \\
\hline Aortic cross-clamp time (min) & $98(76-114)$ & $89(72-119)$ & $112(90-142)$ \\
\hline \multicolumn{4}{|l|}{ Pathogens } \\
\hline Staphylococcus & $54(47.7 \%)$ & $36(40 \%)$ & $18(37.5 \%)$ \\
\hline Other & $11(9.7 \%)$ & $7(7.7 \%)$ & $4(8.3 \%)$ \\
\hline ICU stay (day) & $3(3-7)$ & $2(2-5)$ & $3(3-4)$ \\
\hline LHS (day) & $9(7-14)$ & $8(7-12)$ & $10(7-17)$ \\
\hline Complications & $34(24.6 \%)$ & $22(24.4 \%)$ & $12(25 \%)$ \\
\hline Stroke & $7(5 \%)$ & $5(5.5 \%)$ & $2(4.1 \%)$ \\
\hline${ }^{*} \operatorname{LCOS}$ & $19(13.7 \%)$ & $14(21.1 \%)$ & $5(14.4 \%)$ \\
\hline Re-operation for bleeding & $12(8.6 \%)$ & $8(8.8 \%)$ & $4(10.5 \%)$ \\
\hline
\end{tabular}




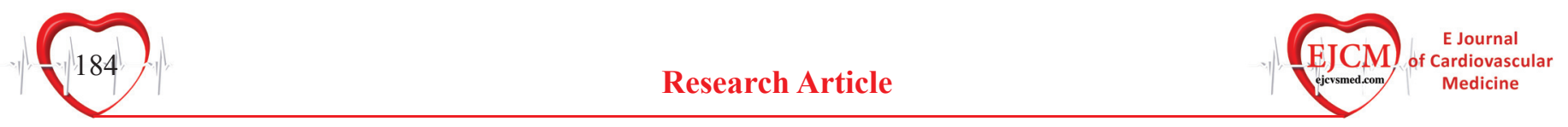

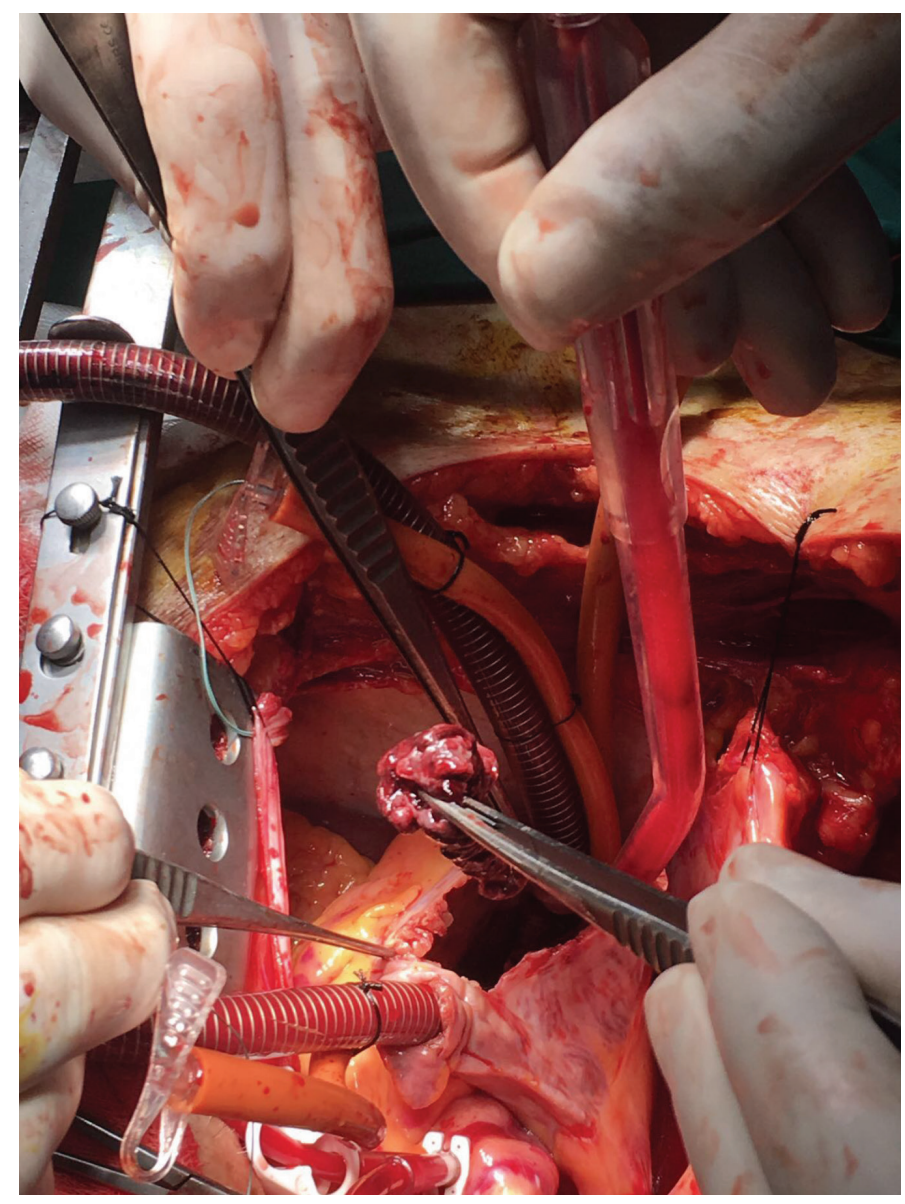

Figure 3. Exhibits the large atrial thrombus related to infective endocarditis in a 19-year-old male patient hospital database or telephone contacts. At the end of 8 years, seven patients required redosurgery for a mild to severe degree of tricuspid valve impairment or a severe paravalvular leak $(\mathrm{n}=2$ in the replacement group vs $\mathrm{n}=5$ in the repair group). Echocardiography showed that there was a high pulmonary artery pressure (median $=56 \pm 13.9$ $\mathrm{mmHg}$ ). In those patients, we performed valve replacement in redo-operations. The patients' status is NYHA Class I-II now.

\section{Discussion}

We presented our clinical experiences of patients with right-sided infective endocarditisis without the involvement of left heart and patients' clinical follow-up. In accordance with previous reports, respiratory symptoms associated with fever, high sedimentation rate, anemia, and dyspnea were predominating symptoms in our case series. Our study showed that patients with congestive heart failure (NYHA Class III-IV), who needed an emergent surgery at the admission to hospital, required significantly higher dose of inotropics and pulmonary support compared to those who underwent elective surgery. These emergent patients may have worsening clinical conditions, longer ICU and hospital stay when compared to those having

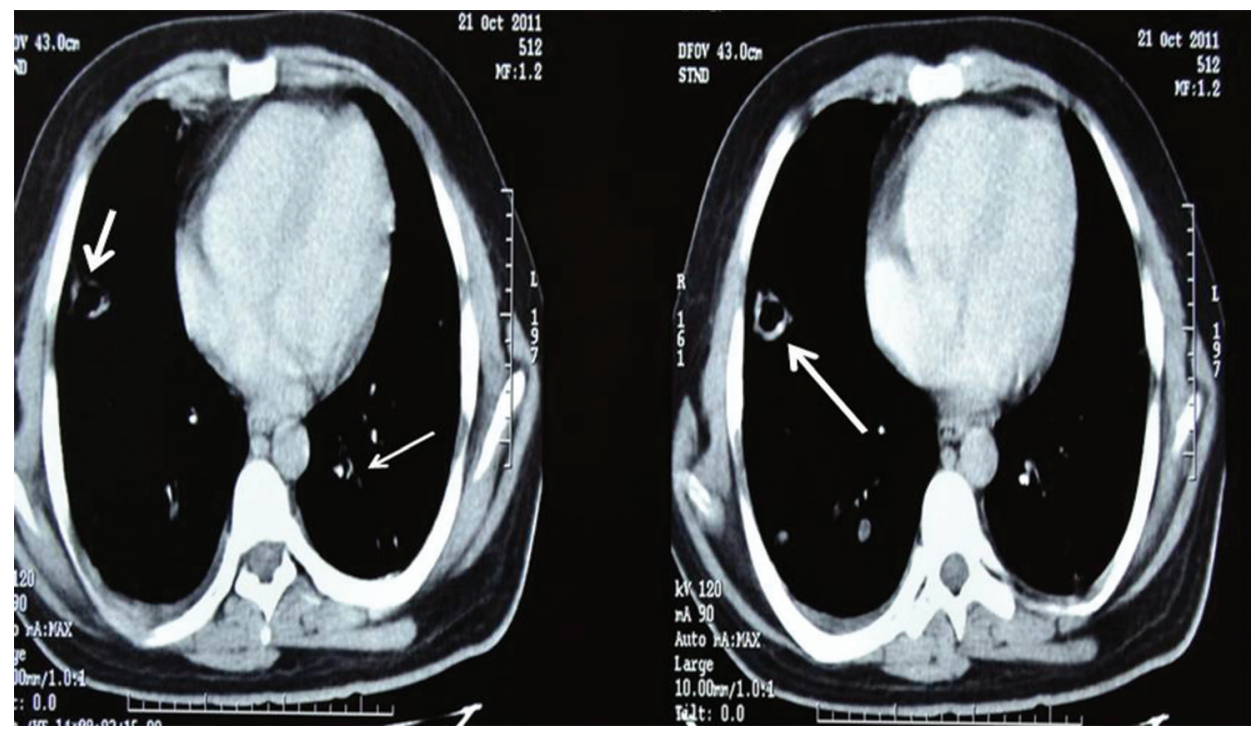

Figure 4. Thoracic computed tomography shows bilateral recurrent pulmonary embolic events in an intravenous drug user patient (White arrows) 
elective surgery. Therefore, early diagnosis of IRSIE and timing of surgery are very important factors for patients' clinical outcomes. We proposed aggressive debridement and valve replacement if there was a peri-annular abscess formation in our series because it might increase morbidity and mortality.

Because IRSIE has no any specific cardiovascular symptoms, its diagnosis may be delayed in some patients. High body temperature, anemia, dyspnea, and pulmonary embolism are the main symptoms which have been reported in $75 \%$ of cases with IRSIE. Therefore, it is difficult to differentiate the symptoms of IRSIE from those of another cardiopulmonary disease such as pneumonitis, intracardiac thrombus, or pulmonary tumor ${ }^{(14,15)}$. In our opinion, to provide patients' survival, the clinicians should keep in mind that IRSIE may be present in patients with nonspecific classical symptoms such as dyspnea, anemia, and a high body temperature.

Seventy percent of the patients with IRSIE are treated conservatively ${ }^{(16)}$. Because there are a small number of cases who are treated surgically, current recommendations of treatment protocols vary ${ }^{(17-19)}$. We operated all patients because of persistent infection despite antibiotic therapy, and patients with recurrent septic pulmonary embolism. Also, we operated about half of our patients with massive tricuspid valve impairment and with heart failure. We performed urgent surgery in patients with IRSIE because of septic shock or congestive heart failure. In a number of patients, the vegetation size was more than $>2 \mathrm{~cm}(\mathrm{n}=39 ; 67.2 \%)$. We detected severe hepatic injury in IVDs that had high alanine aminotransferase and aspartate aminotransferase. We, therefore, suggest early surgery in patients with hepatic failure, Staphylococcus aureus infections or fungi in blood culture, which may deteriorate the patients' condition. Three patients with fungal endocarditis required myocardial and pulmonary support pre-operatively in our series. We have seen that a severe valve damage with the destruction of annular or subannular area or pulmonary embolic events may increase the risk of morbidity and mortality ${ }^{(20-25)}$.
Despite the development in cardiovascular technology, in-hospital mortality rate of IRSIE after surgery is still high $^{(13-17)}$. Unfortunately, the clinical outcomes after surgical procedures of IRSIE remains challenging because of the small amount of publications and patients' heterogeneity. Timing of surgery depends on some factors such as the cause of IRSIEs (pacemaker or ICD lead endocarditis, prosthetic valve endocarditis, infective agents (e.g fungi, Staphylococcus), existence or co-existence with left-sided infective endocarditis, and complications of IRSIE such as intracardiac abscess, large vegetation, or accompanied intracardiac thrombus) $)^{(8,26)}$.

In our patients with IRSIE, who had specific and non-specific cardiopulmonary symptoms, we detected positive blood culture associated with echocardiographic findings such as pulmonary embolic events, leaflet damage including perforation, or intracardiac thrombus. Microorganism could not be detected in some patients $(\mathrm{n}=8 ; 13.7 \%)$. These patients were empirically given antibiotics treatment for more than 10 days. Fungal endocarditis and Enterococcus were detected in seven patients (IVDUs). Itraconazole and caspofungin were suggested as an effective drug of refractory Aspergillus infection in the literature ${ }^{(27)}$. Candida albicans, Aspergillus fumigatus, and Fusarium were the main pathogens in our patients. Considering the previous reports, we used Voriconazole because it had a wide spectrum $^{(28)}$. Amphotericin-B was given at higher doses in the management of Aspergillus because it was less toxic than the conventional amphotericin in patients with kidney dysfunction (dialyses patients) or nephrotoxicity while receiving classic amphotericin.

Chest X-ray and thoracic computed tomography revealed that there were pulmonary embolic events in IVDU. In this group, congestive heart failure and orthopnoea because of recurrent pulmonary embolic events were predominate in their clinical conditions. Early surgical approach was satisfactory in these patients. No mortality was seen after surgery in this group, except for temporary renal failure requiring dialyses. 
Cardiac or pulmonary complications have been reported in $60 \%$ of patients with $\operatorname{IRSIE}^{(29,30)}$. Pulmonary embolic events as a cause of pulmonary infarction or pulmonary abscess as a complication may be seen like in our IVDUs. The dilation of right heart associated with severe tricuspid regurgitation or multiple pulmonary embolus may be detected ${ }^{(31-33)}$.

The authors suggested a different recommendation for timing the surgical approach in these patients ${ }^{(27,28,34)}$. The major indications were persistent infection, recurrent pulmonary embolus, severe tricuspid regurgitation in combination with heart failure, septic shock, and a new onset of renal or hepatic failure. If there are an intracardiac pace-maker lead or dialyses catheter, the authors suggest an early surgery ${ }^{(25,26,29-31)}$. If the size of a vegetation is $>2 \mathrm{~cm}$ despite intensive antibiotic treatment, the authors suggest surgical approach with postoperative intensive antibiotic treatment in the early time ${ }^{(25-27,32,33)}$. Conservative therapy in combination with antibiotics has been proposed as a first choice of treatment method ${ }^{(9,10,11,24,26)} \cdot 20-30 \%$ of patients with IRSIE required surgical approach with an extensive debridement only ${ }^{(9,34-37)}$. However, early surgery has been proposed in order to achieve long-term good clinical outcomes in patients with IRSIE ${ }^{(22-33)}$.

In patients with IRSIE who have concomitant congenital heart disease, the timing of surgery is controversial. Some authors suggested surgery only after fully control of infection ${ }^{(12,21,22,36-39)}$. We proposed an early surgical approach in patients with large size of vegetation in combination with NYHA Class III-IV condition. In our opinion, in patients with large and mobile vegetation, early surgical approach may be provided for pulmonary embolic events and right heart failure.

Our 13 patients with congenital heart disease and with IRSIE (atrial or ventricular septal defect) were in the active phase. In all patients, we repaired intracardiac defect using a fresh pericardium after vegetectomy and valve repair or replacement despite longer ECC and an aortic X-clamp time. All of them recovered successfully after surgery. No infection was detected during follow-up period in these patients. Our principle of the therapeutic approach is the removal of all infected implanted materials such as leads and thrombus, and repair or replacement of cardiac valve after vegetectomy. If there is a severe destruction of valve together with peri-annular abscess, we suggest aggressive debridement and intracardiac biologic material use.

Different techniques of tricuspid valve reconstruction have been suggested according to the degree of valvular damage ${ }^{(32-37)}$. De Vega, Kay's annuloplasty and bicuspidization are the preferred methods. In patients with severe valve damage including peri-annular abscess, valve replacement has been suggested. However, the use of a mechanical or a tissue valve in IRSIE is still a matter of debate ${ }^{(36-42)}$. In the active phase, to provide reinfection, we used a biologic heart valve in our series. To inhibit recurrence of infection, we suggest autologous pericardium for tricuspid valve reconstruction or patient requiring an aggressive debridement of peri-annular abscess formation. In a number of operations, we performed an annuloplasty reinforced with pericardium or ring to ensure leaflet coaptation. In our opinion, these surgical principles provide satisfactory longterm results including avoidance from re-operation. To prevent recurrence of IRSIE, we performed an extensive debridement additionally.

\section{Conclusion}

In the absence of left-sided cardiac infection, early surgery should be considered in patients with IRSIE with cardio-pulmonary complications. If there is an intracardiac abscess and thrombus formation, or recurrent pulmonary embolic events, we suggest early surgery. Patient with mobile and a large vegetation, mycotic endocarditis may need an early surgical approach. Earlier treatment of patients with IRSIE after diagnosis may provide good early, mid- and long-term clinical outcomes with an extensive debridement of vegetations. To prevent recurrences of infection after surgery, we should avoid the use of foreign materials. 


\section{Ethics}

Ethics Committee Approval: Because of its being a retrospective clinical article, we did not receive ethics committee approval.

Informed Consent: Because of the study's retrospective design, no patients' consents were added.

Peer-review: Internally and externally peer-reviewed.

Financial Disclosure: The author declared that this study received no financial support.

\section{References}

1. Hoen B, Alla F, Selton-Suty C, et al. Changing profile of infective endocarditis: results of a 1-year survey in France. JAMA 2002;288:75-81.

2. Cabell $\mathrm{CH}$, Abrutyn E. Progress toward a global understanding of infective endocarditis. Lessons from the International Collaboration on Endocarditis. Cardiol Clin 2003;21:147-58.

3. Miro JM, del Rio A, Mestres CA. Infective endocarditis and cardiac surgery in intravenous drug abusers and HIV-1 infected patients. Cardiol Clin 2003;21:167-84.

4. Musci M, Siniawski H, Pasic M, et al. Surgical treatment of right-sided active infective endocarditis with or without involvement of the left heart: 20-year single center experience. Eur J Cardiothorac Surg 2007;32:118-25.

5. Mylonakis E, Calderwood SB. Infective endocarditis in adults. N Engl $\mathrm{J}$ Med 2001;345:1318-30.

6. Moss R, Munt B. Injection drug use and right sided endocarditis. Heart $2003 ; 89: 577-81$.

7. Nadji G, Remadi JP, Coviaux F, et al. Comparison of clinical and morphological characteristics of Staphylococcus aureus endocarditis with endocarditis caused by other pathogens. Heart 2005;91:932-7.

8. Cook RJ, Ashton RW, Aughenbaugh GL, Ryu JH. Septic pulmonary embolism: presenting features and clinical course of 14 patients. Chest 2005;128:162-6.

9. Elliott TS, Foweraker J, Gould FK, Perry JD, Sandoe JA; Working Party of the British Society for Antimicrobial Chemotherapy. Guidelines for the antibiotic treatment of endocarditis in adults: report of the Working Party of the British Society for Antimicrobial Chemotherapy. J Antimicrob Chemother 2004;54:971-81.

10. Horstkotte D, Follath F, Gutschik E, et al. Guidelines on prevention, diagnosis and treatment of infective endocarditis executive summary; the task force on infective endocarditis of the European Society of Cardiology. Eur Heart J 2004;25:267-76.

11. Moreillon P, Que YA. Infective endocarditis. Lancet 2004;363:139-49.

12. Han L, Zhang BR, Zhu JL, et al. Surgical intervention of right-sided infective endocarditis. Chinese Cardiovasc J 2000;28:452-4.

13. Niwa $K$, Nakazawa $M$, Tateno $S$, Yoshinaga $M$, Terai $M$. Infective endocarditis in congenital heart disease: Japanese national collaboration study. Heart 2005;91:795-800.
14. Darouiche RO. Treatment of infections associated with surgical implants. N Engl J Med 2004;350:1422-9.

15. Wilkoff BL, Byrd CL, Love CJ, et al. Pacemaker lead extraction with the laser sheath: results of the pacing lead extraction with the excimer sheath (PLEXES) trial. J Am Coll Cardiol 1999;33:1671-6.

16. del Rio A, Anguera I, Miro JM, et al. Surgical treatment of pacemaker and defibrillator lead endocarditis: the impact of electrode lead extraction on outcome. Chest 2003;124:1451-9.

17. Moon MR, Miller DC, Moore KA, et al. Treatment of endocarditis with valve replacement: the question of tissue versus mechanical prosthesis. Ann Thorac Surg 2001;71:1164-71.

18. Gottardi R, Bialy J, Devyatko E, et al. Midterm follow-up of tricuspid valve reconstruction due to active infective endocarditis. Ann Thorac Surg 2007;84:1943-8

19. Mestres CA, Castella M, Moreno A, et al. Cryopreserved mitral homograft in the tricuspid position for infective endocarditis: a valve that can be repaired in the long-term (13 years). J Heart Valve Dis 2006;15:389-91.

20. Chan P, Ogilby JD, Segal B. Tricuspid valve endocarditis. Am Heart J 1989:117;1140-6.

21. Frontera JA, Gradon JD. Right-side endocarditis in injection drug users: review of proposed mechanisms of pathogenesis. Clin Infect Dis 2000:30;374-9.

22. Nucifora G, Badano L, Hysko F, Allocca G, Gianfagna P, Fioretti P. Pulmonary embolism and fever: when should right-sided infective endocarditis be considered? Circulation 2007;115:173-9.

23. Pesanti E, Smith IM. Infective endocarditis with negative blood cultures: an analysis of 52 cases. Am J Med 1979;66:43-50.

24. San Roman JA, Vilacosta I. Role of transesophageal echocardiography in right-sided endocarditis. Echocardiography 1995;12:669-72.

25. Margey R, McCann H, Blake G, et al. Contemporary management of and outcomes from cardiac device related infections. Europace 2010;1:6470 .

26. Sohail MR, Uslan DZ, Khan AH, et al. Management and outcome of permanent pacemaker and implantable cardioverter-defibrillator infections. J Am Coll Cardiol 2007;49:1851-9.

27. Gottardi R, Bialy J, Devyatko E, et al. Midterm follow-up of tricuspid valve reconstruction due to active infective endocarditis. Ann Thorac Surg 2007;84:1943-8

28. Head SJ, Mokhles MM, Osnabrugge RL, Bogers AJ, Kappetein AP Surgery in current therapy for infective endocarditis. Vasc Health Risk Manag 2011;7:255-63

29. Uslan DZ, Baddour LM. Cardiac device infections: getting to the heart of the matter. Curr Opin Infect Dis 2006;19:345-8.

30. Cacoub P, Leprince P, Nataf P, et al. Pacemaker infective endocarditis. Am J Cardiol 1998;82:480-4.

31. Lowes JA, Hamer J, Williams G, et al. 10 Years of infective endocarditis at St. Bartholomew's Hospital: analysis of clinical features and treatment in relation to prognosis and mortality. Lancet 1980;1:133-6.

32. Moon MR, Stinson EB, Miller DC. Surgical treatment of endocarditis. Prog Cardiovasc Dis 1997;40:239-64. 
33. Remadi JP, Habib G, Nadji G, et al. Predictors of death and impact of surgery in Staphylococcus aureus infective endocarditis, Ann Thorac Surg 2007;83:1295-302.

34. Chrissoheris MP, Libertin C, Ali RG, Ghantous A, Bekui A, Donohue T. Endocarditis complicating central venous catheter bloodstream infections: a unique form of health care associated endocarditis. Clin Cardiol 2009;32:48-54.

35. Morokuma H, Minato N, Kamohara K, MinematsuN. Three surgical cases of isolated tricuspid valve infective endocarditis. Ann Thorac Cardiovasc Surg 2010;16:134-8.

36. Musci M, Siniawski H, Pasic M, et al. Surgical treatment of right-sided active infective endocarditis with or without involvement of the left heart: 20-year single center experience. Eur J Cardiothorac Surg 2007;32:11825.

37. Hecht SR, Berger M. Right-sided endocarditis in intravenous drug users: prognostic features in 102 episodes. Ann Intern Med 1992;117:560-6.
38. Revilla A, López J, Villacorta E, et al. Isolated right-sided valvular endocarditis in non-intravenous drug users. Rev Esp Cardiol 2008;61:12539.

39. Habib G, Hoen B, Tornos P, et al. Guidelines on the 40. Prevention, Diagnosis, and Treatment of Infective Endocarditis (new version 2009): the Task Force on the Prevention, Diagnosis, and Treatment of Infective Endocarditis of the European Society of Cardiology (ESC), Eur Heart J 2009;30:2369-413.

40. Lange R, De Simone R, Bauernschmitt R, TanzeemA, Schmidt C, Hagl $\mathrm{S}$. Tricuspid valve reconstruction, a treatment option in acute endocarditis, Eur J Cardiothorac Surg 1996;10:320-6.

41. Chen $\mathrm{C}$, Huang $\mathrm{CH}$, Chen YC. Timing of surgery for fungal infective endocarditis. Heart Surg Forum 2017;20:129-31.

42. Fayad G, Modine T, Lions C, et al. Conservative surgical treatment for active infective tricuspid valve endocarditis according to the "clover technique". Heart Surg Forum 2008;11:E120-6. 